ARTICLE

https://doi.org/10.1038/s41467-019-13580-w

\title{
Efficient sky-blue perovskite light-emitting diodes via photoluminescence enhancement
}

Qi Wang ${ }^{1}$, Xiaoming Wang ${ }^{2}$, Zhi Yang (10 ${ }^{3}$, Ninghao Zhou ${ }^{4}$, Yehao Deng $\mathbb{D}^{1}{ }^{1}$, Jingjing Zhao ${ }^{1}$, Xun Xiao (D) ${ }^{1}$, Peter Rudd ${ }^{1}$, Andrew Moran ${ }^{4}$, Yanfa Yan (D) ${ }^{2} \&$ Jinsong Huang (iD ${ }^{1 \star}$

The efficiencies of green and red perovskite light-emitting diodes (PeLEDs) have been increased close to their theoretical upper limit, while the efficiency of blue PeLEDs is lagging far behind. Here we report enhancing the efficiency of sky-blue PeLEDs by overcoming a major hurdle of low photoluminescence quantum efficiency in wide-bandgap perovskites. Blending phenylethylammonium chloride into cesium lead halide perovskites yields a mixture of two-dimensional and three-dimensional perovskites, which enhances photoluminescence quantum efficiency from $1.1 \%$ to $19.8 \%$. Adding yttrium (III) chloride into the mixture further enhances photoluminescence quantum efficiency to $49.7 \%$. Yttrium is found to incorporate into the three-dimensional perovskite grain, while it is still rich at grain boundaries and surfaces. The yttrium on grain surface increases the bandgap of grain shell, which confines the charge carriers inside grains for efficient radiative recombination. Record efficiencies of $11.0 \%$ and $4.8 \%$ were obtained in sky-blue and blue PeLEDs, respectively.

\footnotetext{
${ }^{1}$ Department of Applied Physical Sciences, University of North Carolina at Chapel Hill, Chapel Hill, North Carolina 27599, USA. ${ }^{2}$ Department of Physics and Astronomy and Wright Center for Photovoltaics Innovation and Commercialization, The University of Toledo, Toledo, Ohio 43606, USA. ${ }^{3}$ Electronic Materials Research Laboratory, Key Laboratory of Education Ministry, International Center for Dielectric Research, Xi'an Jiaotong University, Xi'an 710049, China. ${ }^{4}$ Department of Chemistry, University of North Carolina at Chapel Hill, Chapel Hill, North Carolina 27599, USA. *email: jhuang@unc.edu
} 
erovskite light-emitting diodes (PeLEDs) have drawn tremendous research interest in recent years due to the attractive properties of metal halide perovskite materials such as earth abundance, solution process capability, large carrier mobility, and excellent color purity ${ }^{1-12}$. The versatile bandgap tuning of perovskite materials $\left(\mathrm{ABX}_{3}\right)$ via composition tuning of $\mathrm{A}, \mathrm{B}$, and $\mathrm{X}$ sites makes them applicable in whole visible light range. The application of all inorganic cesium-based perovskite significantly enhances the material stability under electric field ${ }^{13,14}$. Very impressive progresses have been made in enhancing the efficiencies of perovskite LEDs in the past few years ${ }^{3-12}$. External quantum efficiencies (EQEs) approaching 20\% have been reported for both green and red PeLEDs, which ravel those from optimized organic phosphorescence light-emitting diodes $(\text { LEDs })^{3-9}$. However, blue-emission PeLEDs, which are important for display and lighting applications, have efficiency much lower than green and red PeLEDs. The highest reported EQE for skyblue PeLEDs is still $<10 \%{ }^{15-18}$. One convenient way to make blue-emission perovskite films is to incorporate chlorine $(\mathrm{Cl})$ into Br-based perovskites, whereas one critical issue for the $\mathrm{Cl}: \mathrm{Br}$ mixed or pure Cl-based perovskite films is their small photoluminescence quantum efficiency (PLQE) below 20\%, which is much lower than the green- or red-emission perovskite films with a typical PLQE of $70^{3-9,19,20}$. As only about a quarter of light could be extracted out of PeLED devices made on glass substrates due to the mismatched refractive index ${ }^{21}$, the highest EQE for the blue devices will be limited to $<5 \%$, assuming perfect charge balance can be achieved in the devices. The exact reason for the low PLQEs in chlorinated perovskite films is not clear. One way used in previous studies to increase PLQE was adopting quantum dots or two-dimensional (2D) layered perovskites, in which strong quantum confinement could significantly improve the film PLQE to over $80 \%^{22}$. However, due to the inferior charge transport in these films, high EQE sky-blue PeLEDs are still rarely reported.

In this study, we report significantly enhanced PLQE of skyblue-emission polycrystalline perovskite films from $1.1 \%$ to $49.7 \%$ by incorporating $\mathrm{PEACl}$ and $\mathrm{YCl}_{3}$ into three-dimensional (3D) $\mathrm{Cs} \mathrm{PbBr}_{3}$ perovskite films, where $\mathrm{PEACl}$ is phenylethylammonium chloride $\left(\mathrm{C}_{6} \mathrm{H}_{5} \mathrm{C}_{2} \mathrm{H}_{4} \mathrm{NH}_{3} \mathrm{Cl}\right)$ and $\mathrm{YCl}_{3}$ is Yttrium (III) chloride. The sky-blue PeLEDs with $2 \% \mathrm{YCl}_{3}$ showed high EQE of $11.0 \%$ and maximum brightness of $9040 \mathrm{~cd} \mathrm{~m}^{-2}$. Blue PeLED with $4.8 \%$ EQE was obtained by adding $10 \% \mathrm{YCl}_{3}$ in the perovskite film, with a device Commission Internationale de l'Eclairage (CIE) coordinate of $(0.10,0.13)$.

\section{Results}

Photoluminescence enhancement by $\mathrm{YCl}_{3}$ in perovskite films. We fabricated and studied perovskite films with two different compositions in this study: $3 \mathrm{D}$ CsPbBr${ }_{2.4} \mathrm{Cl}_{0.6}$ film and $2 \mathrm{D}-3 \mathrm{D}$ mixed $\mathrm{CsPbBr}_{3}: \mathrm{PEACl}$ film (molar ratio is $1: 1$ in precursor solution). Details about the film fabrication could be found in the Methods section. Chlorine was added in these films so that both films emit sky-blue light, with photoluminescence (PL) peaks at $486 \mathrm{~nm}$ for $\mathrm{CsPbBr}_{2.4} \mathrm{Cl}_{0.6}$ film and $487 \mathrm{~nm}$ for $\mathrm{CsPbBr}_{3}: \mathrm{PEACl}$ (1:1) film (Fig. 1a). The corresponding CIE coordinates are $(0.06$, $0.26)$ and $(0.08,0.25)$, respectively (Fig. 1b). Under the ultraviolet (UV) lamp, CsPbBr 3 :PEACl (1:1) film exhibits much brighter PL emission than the $\mathrm{CsPbBr}_{2.4} \mathrm{Cl}_{0.6}$ film (Fig.1c). Figure 1d shows PLQEs of $\mathrm{CsPbBr}_{2.4} \mathrm{Cl}_{0.6}$ film and $\mathrm{CsPbBr}_{3}: \mathrm{PEACl}$ (1:1) film under different incident laser intensities. Maximum PLQE of $\mathrm{CsPbBr}_{3}: \mathrm{PEACl}(1: 1)$ film (19.8\%) is much higher than that of
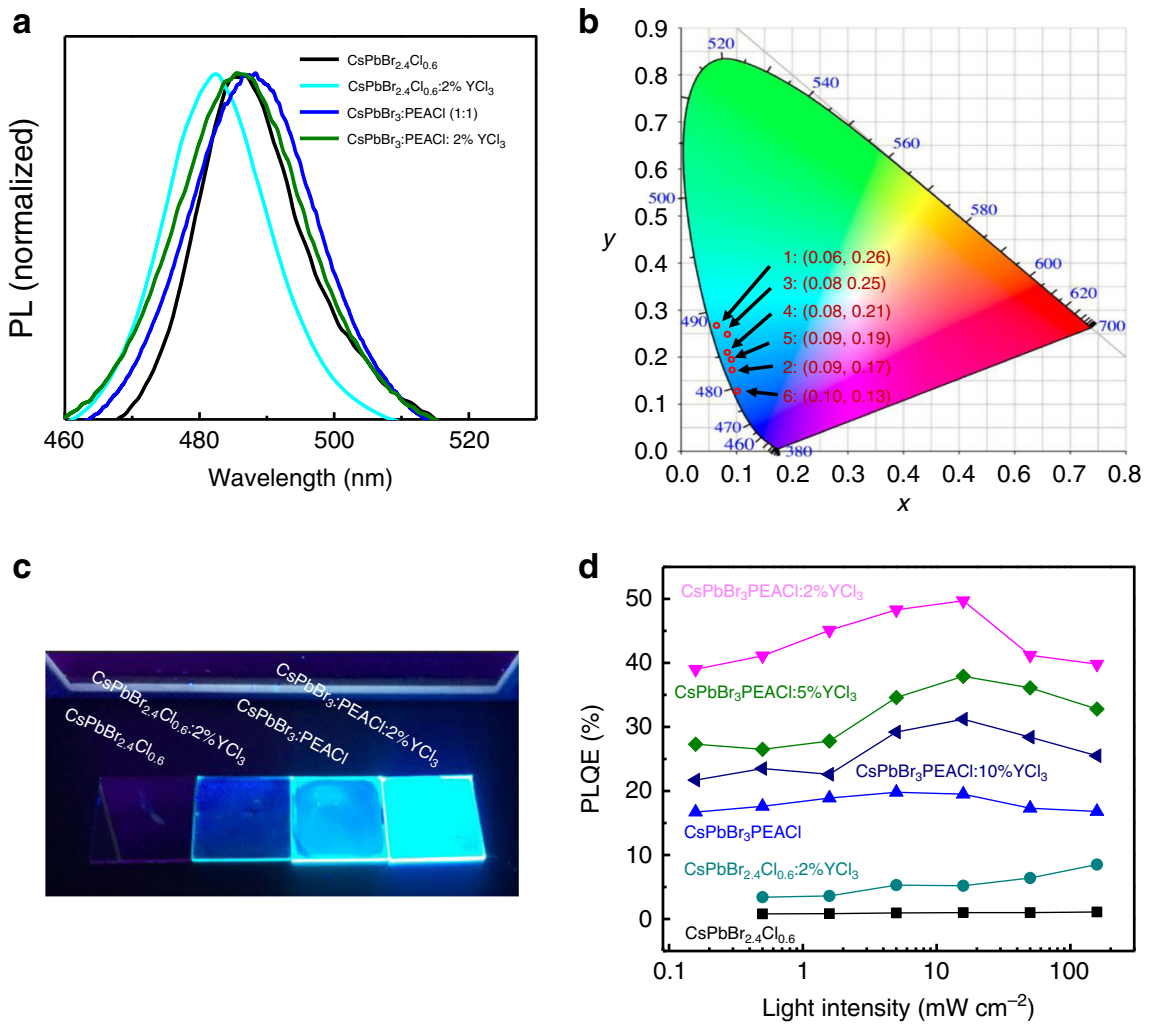

Fig. 1 Photoluminescence enhancement by $\mathbf{P E A C l}$ and $\mathbf{Y C l}_{\mathbf{3}}$ incorporation in perovskite films. a $\mathrm{PL}$ spectra of $\mathrm{CsPbBr}_{2.4} \mathrm{Cl}_{0.6}, \mathrm{CsPbBr}_{2.4} \mathrm{Cl}_{0.6}: 2 \% \mathrm{YCl}_{3}$, $\mathrm{CsPbBr}_{3}: \mathrm{PEACl}(1: 1)$, and $\mathrm{CsPbBr}_{3}: \mathrm{PEACl}: 2 \% \mathrm{YCl}_{3}$ films. b ClE coordinates of $\mathrm{CsPbBr}_{2.4} \mathrm{Cl}_{0.6}$ (1), $\mathrm{CsPbBr}_{2.4} \mathrm{Cl}_{0.6}: 2 \% \mathrm{YCl}_{3}(2), \mathrm{CsPbBr}_{3}: \mathrm{PEACl}(1: 1)(3)$, and $\mathrm{CsPbBr}_{3}: \mathrm{PEACl}: 2 \% \mathrm{YCl}_{3}$ (4) films. ClE coordinates of $\mathrm{CsPbBr}_{3}: \mathrm{PEACl}_{2} \% \mathrm{YCl}_{3}$ (5) device and $\mathrm{CsPbBr}_{3}: \mathrm{PEACl}_{10} \% \mathrm{YCl}_{3}$ (6) device. c $\mathrm{A}$ photograph of the films under UV lamp. The composition of each film is labeled in the photograph. d Power-dependent PLQEs of the perovskite films with different compositions. 
$\mathrm{CsPbBr}_{2.4} \mathrm{Cl}_{0.6}$ film (1.1\%). Phenylethylammonium halide incorporation in $3 \mathrm{D}$ perovskites usually introduces layered phases in the films, which has been demonstrated as an effective method to increase the PLQEs of green perovskite films ${ }^{23,24}$. Therefore, we focused on $\mathrm{CsPbBr}_{3}: \mathrm{PEACl}(1: 1)$ perovskite in this study because of its higher PLQE compared with $3 \mathrm{D} \mathrm{CsPbBr}_{2.4} \mathrm{Cl}_{0.6}$ film. PL spectra of perovskite films with different $\mathrm{CsPbBr}_{3}: \mathrm{PEACl}$ ratios are shown in Supplementary Fig. 1, which shows blue shift of PL peak from $522 \mathrm{~nm}$ for $\mathrm{CsPbBr}_{3}$ film to $487 \mathrm{~nm}$ for $\mathrm{CsPbBr}$ : PEACl (1:1). Further increasing PEACl ratio in the films to $\mathrm{CsPbBr}_{3}: \mathrm{PEACl}(1: 2)$ results in a much lower PL intensity and very broad emission band with multiple peaks, which might be from self-trapped exciton emission of different layered phases ${ }^{25}$. $\mathrm{X}$-ray diffraction (XRD) study of the $\mathrm{CsPbBr}_{3}: \mathrm{PEACl}(1: 2)$ film in Supplementary Fig. 2 clearly shows more layered phases are formed with the increased $\mathrm{PEACl}$ ratio in the films.

The PLQE of $19.8 \%$ in $\mathrm{CsPbBr}_{3}$ :PEACl (1:1) film still limits the EQE of sky-blue PeLED to be $<5 \%$, considering a typical outcoupling efficiency around $20 \%$ in perovskite LEDs ${ }^{21}$. Encouragingly, we found that adding $2 \% \mathrm{YCl}_{3}$ in the $\mathrm{CsPbrr}_{3}: \mathrm{PEACl}(1: 1)$ film further increased PLQE to $49.7 \%$, as shown in Fig. 1d. Here, the yttrium ratio is defined as the molar ratio of $\mathrm{Y}$ to $\mathrm{Pb}$ in the precursor solution. The photographs in Fig. 1c and Supplementary Fig. 3 clearly shows the PEACl:CsPbBr 3 (1:1) films with $\mathrm{YCl}_{3}$ have much stronger PL emission than the film without $\mathrm{YCl}_{3}$. The maximum PLQEs of PEACl:CsPbBr 3 (1:1) films with 2\%, 5\%, and $10 \% \mathrm{YCl}_{3}$ are $49.7 \%, 37.9 \%$, and $31.2 \%$, respectively, which are much higher than the film without $\mathrm{YCl}_{3}$. Adding $\mathrm{YCl}_{3}$ in the PEACl:CsPbBr ${ }_{3}$ (1:1) film also blue shifts the PL peak (Supplementary Fig. 3). PEACl:CsPbBr 3 (1:1) films with 2\%, $5 \%$, and $10 \% \mathrm{YCl}_{3}$ exhibit PL peak at 485,483 , and $477 \mathrm{~nm}$, respectively. The decreased PLQE in $5 \%$ and $10 \% \mathrm{YCl}_{3}$ perovskite films compared with $2 \% \mathrm{YCl}_{3}$ perovskite film might be caused by the formation of $\mathrm{YCl}_{3}$ clusters, which may hinder perovskite grain growth (Supplementary Fig. 4). Adding $2 \% \mathrm{YCl}_{3}$ in the $3 \mathrm{D}$ $\mathrm{Cs} \mathrm{PbBr}_{2.4} \mathrm{Cl}_{0.6}$ film also significantly increases the film PLQE from $1.1 \%$ to $8.5 \%$, as shown in Fig. 1d. The photographs in Supplementary Fig. 5 illustrate the PL of $\mathrm{CsPbBr}_{3}$ film was significantly improved by $\mathrm{YBr}_{3}$ treatment.

Efficiency and stability of sky-blue PeLEDs with $\mathbf{Y C l}_{3}$. We fabricated PeLEDs with the device structure shown in Fig. 2a to evaluate the impact of the PLQE enhancement on device efficiency. $\mathrm{PEACl}_{\mathrm{Cs}} \mathrm{PbBr}_{3}$ (1:1) perovskite films with different amount of $\mathrm{YCl}_{3}$ were fabricated between hole transporting layer (HTL) poly(3,4-ethylenedioxythiophene) polystyrene sulfonate (PEDOT:PSS), and electron transporting layer 2,2',2"-(1,3,5Benzinetriyl)- tris(1-phenyl-1-H-benzimidazole) (TPBI). A photo of an operating sky-blue PeLED is shown in Fig. 2b, which shows bright sky-blue emission. Figure 2c-e show current density $(J)$ bias $(V)$, luminance $(B)$-bias $(V)$, and EQEs curves of the PEACl: $\mathrm{CsPbBr} 3$ (1:1) devices with different ratios of $\mathrm{YCl}_{3}$. It was found that adding PEACl in the perovskite film improved the film morphology by forming pinhole-free and smooth film, as shown by the scanning electron microscopy (SEM) images in Supplementary Fig. 4. The pinhole free perovskite film reduced leakage current and improve efficiency in the device, as $\mathrm{CsPBr}_{3}$ device showed large current leakage (Fig. 2c-e). The device with PEACl: $\mathrm{CsPbBr}_{3}$ (1:1) has an EQE of $5.6 \%$ and maximum brightness of $5183 \mathrm{~cd} \mathrm{~m}^{-2}$. Adding $2 \% \mathrm{YCl}_{3}$ in the perovskite film significantly increases $\mathrm{EQE}$ and maximum luminance to $11.0 \%$ and $9040 \mathrm{~cd} \mathrm{~m}^{-2}$, respectively. Figure $2 \mathrm{f}$ shows statistic EQEs of PEACl:CsPbBr ${ }_{3}$ (1:1) devices without and with $2 \% \mathrm{YCl}_{3}$, which clearly demonstrates the efficiency enhancement by adding $\mathrm{YCl}_{3}$ in the devices. Angular emission intensity of the PeLEDs follows a
Lambertian profile, as shown in Supplementary Fig. 6. Further increasing the $\mathrm{YCl}_{3}$ ratio to $5 \%$ and $10 \%$ reduced device EQEs to $8.7 \%$ and $4.8 \%$, respectively. The device with $10 \% \mathrm{YCl}_{3}$ shows EL peak at $477 \mathrm{~nm}$ with a CIE coordinate of $(0.10,0.13)$, which comes to blue light region, as shown in Fig. $1 \mathrm{~b}$ and Supplementary Fig. 7. The EQE variation of devices with different $\mathrm{YCl}_{3}$ ratios closely follows the trend of PLQE change in Fig. 1d, indicating the limiting factor for the efficiency of these sky-blue-emission PeLEDs is the PLQE of the perovskite films, whereas the electron and hole current has been well balanced through controlling the thickness of the charge transport layers in this work (Supplementary Figs. 8 and 9).

It has been widely reported that mixed-halide perovskites, such as $\mathrm{CsPb}\left(\mathrm{Br}_{0.5} \mathrm{Cl}_{0.5}\right)_{3}$, undergo a quick phase segregation under electric field, caused by charge-enhanced ion migration ${ }^{22,26-29}$. Such phase segregation can generate bromine-rich domains and chlorine-rich domains in $\mathrm{CsPb}\left(\mathrm{Br}_{0.5} \mathrm{Cl}_{0.5}\right)_{3}$ films, resulting in severe change of electroluminescent (EL) spectrum during the operation of PeLEDs ${ }^{22,26-29}$. We tested the EL spectrum and luminance stabilities of the most efficient sky-blue PeLEDs to evaluate whether such a phase segregation occurs in these mixed perovskite films. In striking contrast, we found the EL spectra are very stable for the PEACl:CsPbBr ${ }_{3}$ (1:1) LEDs with $\mathrm{YCl}_{3}$. Figure $2 \mathrm{~g}$ shows the EL emission spectra of the sky-blue PeLED under constant bias of $3.2 \mathrm{~V}$ for $120 \mathrm{~min}$. The device shows a stable EL emission with a peak at $485 \mathrm{~nm}$. Supplementary Fig. 10 also shows negligible EL spectrum variation of the device under varied biases. The EL luminance stability of PeLEDs was also tested by fixing the applied voltage bias of $3.2 \mathrm{~V}$, with an initial luminance around $100 \mathrm{~cd} \mathrm{~m}^{-2}$, as shown in Fig. $2 \mathrm{~h}$.

The significantly improved spectrum stability in PEACl: $\mathrm{CsPbBr}_{3}$ (1:1) films with $2 \% \mathrm{YCl}_{3}$ compared with $3 \mathrm{D}$ perovskite materials was ascribed to the dramatically suppressed ion migration in these films, as phase segregation occurs via ion migration $^{28,30}$. To verify it, we studied the activation energies of ionic conduction in both $\mathrm{CsPbBr}{ }_{2.4} \mathrm{Cl}_{0.6}$ and $\mathrm{PEACl}: \mathrm{CsPbBr}_{3}(1: 1)$ films using an established method ${ }^{31,32}$. As shown in Fig. $2 \mathrm{i}$, the ion conduction activation energy of $3 \mathrm{D} \mathrm{CsPbBr}{ }_{2.4} \mathrm{Cl}_{0.6}$ film in the dark is around $0.26 \mathrm{eV}$, which is consistent with previously reported values ${ }^{33}$. In striking contrast, we found that ionic conduction is negligible in the $\mathrm{PEACl}: \mathrm{CsPbr}_{3}(1: 1)$ film in the temperature range up to $350 \mathrm{~K}$. The derived small activation energy of $0.04 \mathrm{eV}$ can be assigned to electronic conduction. This indicates that the ion migration in the $\operatorname{PEACl}_{\mathrm{CsPbBr}}(1: 1)$ films is significantly suppressed by the presence of layered perovskites, consistent with a previous study ${ }^{34,35}$. In addition, adding $2 \% \mathrm{YCl}_{3}$ in $3 \mathrm{D} \mathrm{CsPbBr}{ }_{2.4} \mathrm{Cl}_{0.6}$ film also increased ion migration activation energy from $0.26 \mathrm{eV}$ to $0.75 \mathrm{eV}$.

Phase composition study in $\mathrm{CsPbBr}_{3}$ :PEACl (1:1) film. To understand the role of $\mathrm{PEACl}$, we studied phase composition of the PEACl:CsPbBr $\mathrm{Pb}_{3}$ (1:1) film by performing XRD measurements.

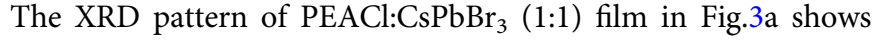
mixed phases of $n=1, n=2$, and $n \geq 3$ perovskites, and each peak is indexed. Most XRD peaks of the PEACl:CsPbBr ${ }_{3}$ (1:1) film can be assigned to $n=1$ phase, based on reported XRD pattern of $(\mathrm{PEA})_{2} \mathrm{PbBr}_{4}$ single crystal ${ }^{36}$. The XRD peak intensity of the $n=1$ phase is much stronger than the other phases, indicating $n=1$ layered phase may prefer to lay down in parallel to the substrate. The $\mathrm{Cl}: \mathrm{Br}$ ratio in $n=1$ layered phase is estimated to be 1:4, based on the XRD and PL peak positions, as shown in Supplementary Figs. 11 and 12. The XRD peak at $4.3^{\circ}$ is most likely from $n=2$ phase, based on the analysis of lattice constants. The XRD diffraction peak of $n \geq 3$ phase locates at a larger diffraction angle of $31.1^{\circ}$ than (202) plane of $\mathrm{CsPbBr}$ 
a

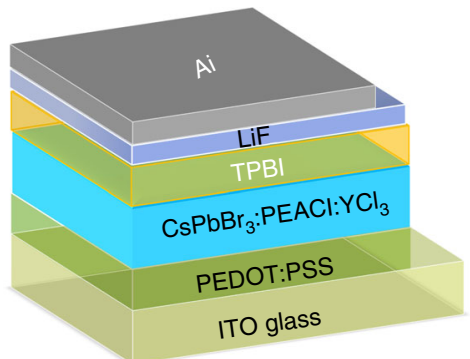

b

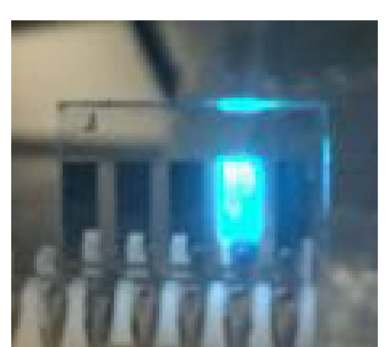

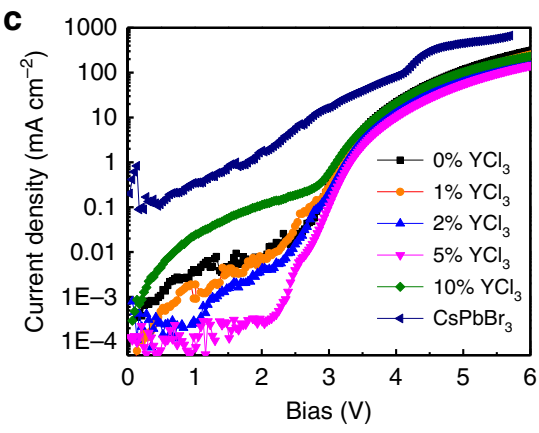

c

f

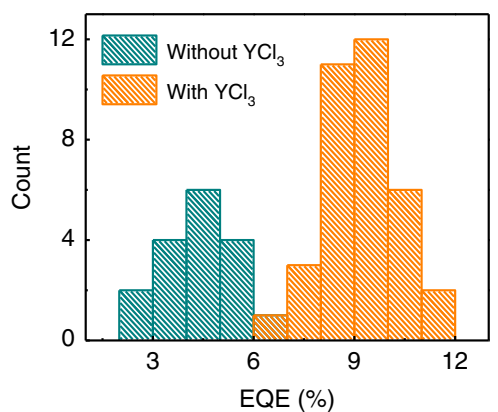

g

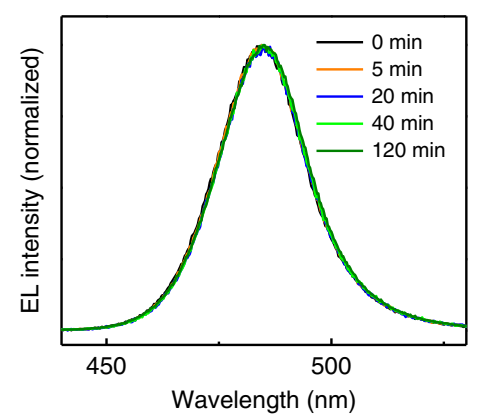

e

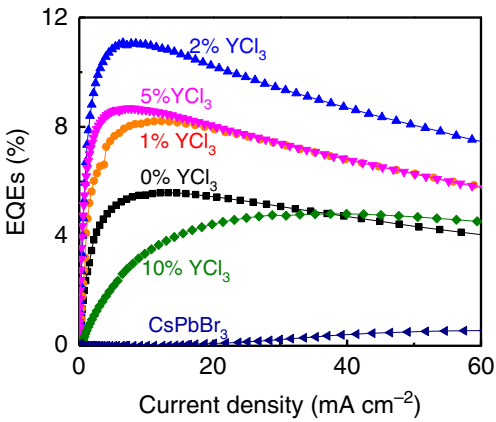

h

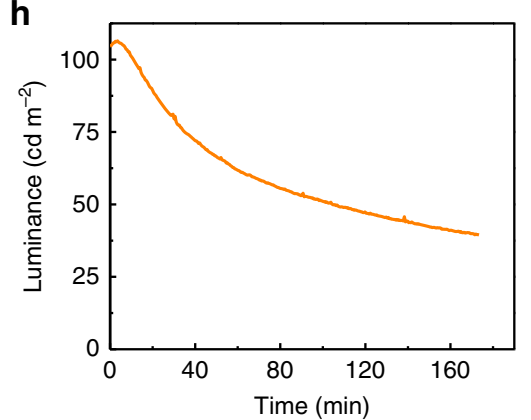

i

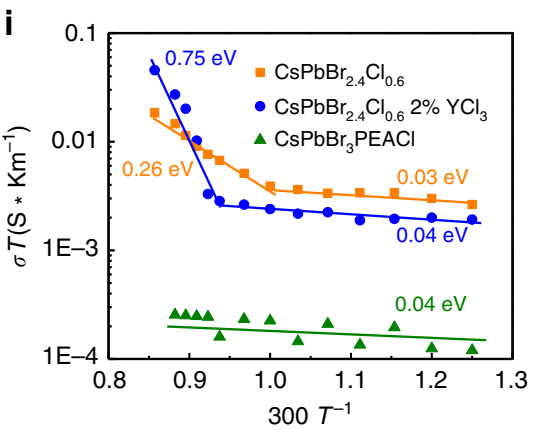

Fig. 2 Efficiency and stability of sky-blue PeLEDs with $\mathbf{Y C l}_{\mathbf{3}}$. a Device structure of the PeLEDs. $\mathbf{b}$ A photograph of the fabricated PeLED showing sky-blue EL emission under bias. Current density-bias (c), luminance-bias (d), EQE-current density (e) curves of $\mathrm{CsPbBr}_{3}: \mathrm{PEACl}(1: 1)$ devices with different ratios of $\mathrm{YCl}_{3}$. Current density-bias (c), luminance-bias (d), EQE-current density (e) curves of a $\mathrm{CsPbBr}_{3}$ perovskite LED. $\mathbf{f}$ Statistic EQEs of sky-blue PeLEDs with or without $\mathrm{YCl}_{3}$. $\mathbf{g}$ Electroluminescence spectrum stability test of a sky-blue PeLED with continuous bias of $3.2 \mathrm{~V}$ for $120 \mathrm{~min}$. $\mathbf{h}$ Operational stability test of a sky-blue PeLED with initial luminance around $100 \mathrm{~cd} \mathrm{~m}^{-2}$. The stability test was conducted in $\mathrm{N}_{2}$ glovebox without device encapsulation. $\mathbf{i}$ Temperature-dependent conductivity measurements to reveal the ion migration activation energies of $\mathrm{CsPbBr}_{2.4} \mathrm{Cl}_{0.6}, \mathrm{CsPbBr}_{2.4} \mathrm{Cl}_{0.6}$ with $2 \% \mathrm{YCl}_{3}$, and $\mathrm{CsPbBr}$ : $\mathrm{PEACl}(1: 1)$ films.

$\left(30.7^{\circ}\right)$, whereas it is very close to the (202) plane of $3 \mathrm{D}$ perovskite with a composition of $\mathrm{CsPbBr}{ }_{2.4} \mathrm{Cl}_{0.6}$, as shown in Supplementary Fig. 13. Hereinafter, we refer the $n \geq 3$ phase in the PEACl: $\mathrm{CsPbBr}_{3}$ (1:1) film as a 3D phase. As shown in Fig. 3b, the PL spectrum from $\mathrm{PEACl}_{\mathrm{Cs}} \mathrm{PbBr}_{3}$ (1:1) film at room temperature only has one emission peak around $489 \mathrm{~nm}$. Reducing the temperature to $173 \mathrm{~K}$ leads to two additional peaks in the PL spectrum at $392 \mathrm{~nm}$ and $419 \mathrm{~nm}$, which are most likely from $n=1$ and $n=2$ phases, respectively ${ }^{37}$. The PL emission around $490 \mathrm{~nm}$ in the $\mathrm{PEACl}: \mathrm{CsPBr}_{3}$ (1:1) perovskite films should be from the $3 \mathrm{D}$ phase with a composition approximately $\mathrm{Cs} \mathrm{PbBr}_{2.4} \mathrm{Cl}_{0.6}$. The absorbance spectrum of PEACl:CsPbBr 3 (1:1) film in Fig. 3c also shows two obvious peaks at $390 \mathrm{~nm}$ and $418 \mathrm{~nm}$, which are close to the PL emission peaks of $n=1$ and $n=2$ phases, respectively.

As the PL emission from the PEACl:CsPbBr ${ }_{3}$ (1:1) perovskite film at room temperature has only one peak from the $3 \mathrm{D}$ phase, there should be efficient energy transfer from layered perovskites to $3 \mathrm{D}$ perovskite. To verify it, we performed transient absorption measurement to study the charge-carrier dynamics in PEACl: $\mathrm{CsPbBr}_{3}$ (1:1) perovskite films. As shown in Fig. 3d, the bleach peak around $485 \mathrm{~nm}$ belongs to the $3 \mathrm{D}$ phase in the PEACl: $\mathrm{CsPbBr}_{3}$ (1:1) film. The bleach peak evolved in the first 2 ps after light excitation, suggesting a fast energy transfer from layered perovskites to $3 \mathrm{D}$ phase (Fig. 3e). After 2 ps, the energy funneling is complete, followed by charge recombination in $3 \mathrm{D}$ perovskite. The energy transfer from layered phases to $3 \mathrm{D}$ phase functions as an energy and carrier concentrator to enhance radiative recombination efficiency for higher PLQE, which has been reported in red-emission $2 \mathrm{D}-3 \mathrm{D}$ mixed perovskite films ${ }^{38}$. The promoted radiative recombination should give rise to higher PLQE observed in the PEACl:CsPbBr ${ }_{3}$ (1:1) perovskite films.

Mechanism of PLQE enhancement by $\mathbf{Y C l}_{3}$. We investigated the distribution of yttrium in the $\mathrm{CsPbBr}{ }_{2.4} \mathrm{Cl}_{0.6}$ and $\mathrm{PEACl}: \mathrm{Cs} \mathrm{PbBr}_{3}$ (1:1) films, to understand its functions in PLQE enhancement. We first examined whether yttrium ions incorporated into the $3 \mathrm{D}$ perovskite phase. We grew single crystals of $\mathrm{CsPbBr}_{3}$ with the presence of $\mathrm{YCl}_{3}$ in the solution. The $\mathrm{Y}$ distribution in the crystal was studied by inductively coupled plasma (ICP) mass spectrometry, which is a sensitive technique to detect trace metal ions as 


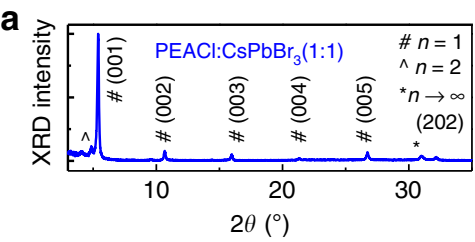

C
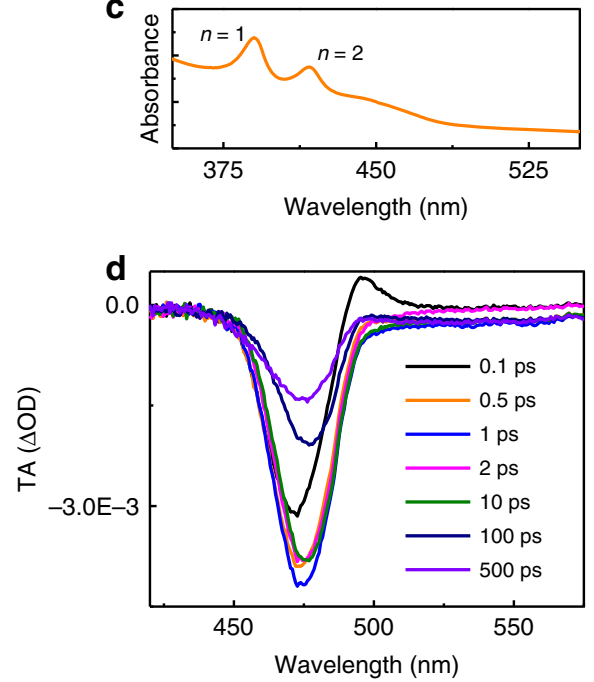

b
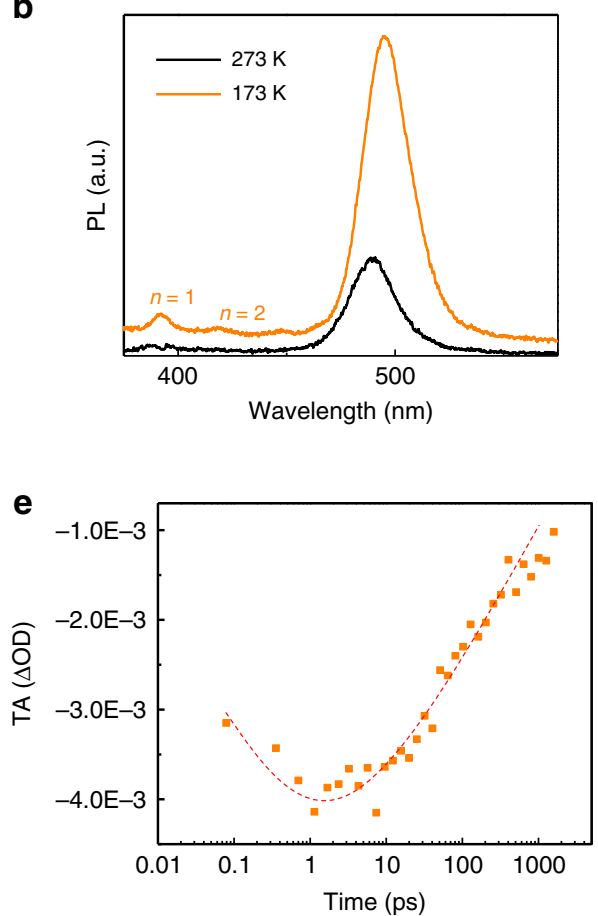

Fig. 3 Phase composition study of $\mathbf{C s P b B r}_{3}: \mathbf{P E A C l}(\mathbf{1 : 1 )}$ film and the function of layered phases. a XRD of $\mathrm{CsPbBr}$ : $: \mathrm{PEACl}(1: 1)$ film. $\mathbf{b} \mathrm{PL}$ spectra of $\mathrm{CsPbBr}_{3}: \mathrm{PEACl}$ (1:1) film at $273 \mathrm{~K}$ and $173 \mathrm{~K}$. c Absorbance of $\mathrm{CsPbBr}_{3}: \mathrm{PEACl}$ (1:1) film. d Transient absorption (TA) spectra of CsPbBr $3: \mathrm{PEACl}(1: 1)$ film at different timescales. e TA signal measured at a probe wavelength of $480 \mathrm{~nm}$ reveals distinct bleaching of the $3 \mathrm{D}$ phase in the $\mathrm{CsPbBr}: \mathrm{PEACL}(1: 1)$ film.

low as particle per quadrillion. The details about crystal growth can be found in the Methods section ${ }^{39,40}$. The single crystal was sliced into small pieces and the compositions of the crystal at different depth were measured. As shown in Fig. $4 \mathrm{a}$, the $\mathrm{Y} / \mathrm{Pb}$ ratio in the crystal center is $0.5 \%$, demonstrating yttrium ions could incorporate into $\mathrm{CsPbBr}_{3}$ perovskite crystals. In addition, it was interesting to find the $\mathrm{Y} / \mathrm{Pb}$ ratio gradually increase from the crystal center to surface, yielding an yttrium concentration gradient in the crystal. A gradient distribution of $\mathrm{Y}$ ions can be explained by the enhanced chemical pressure in the solution during the growth of crystal, because more $\mathrm{Y}$ ions excluded into solution during crystal growth increase its concentration in solution. Similar to single crystal growth, the larger amount of $Y$ added in solution for thin film growth should also cause a concentration gradient, as illustrated in Fig. 4b. Absorption and PL study on thin films in Fig. 4c, d revealed the bandgap of $\mathrm{CsPbBr}_{3}$ was increased by $\mathrm{Y}$ incorporation. We also found a high density of yttrium on the perovskite film surface using surface-sensitive $\mathrm{X}$-ray photoelectron spectroscopy (XPS) measurements. The XPS spectra of PEACl:CsPbBr 3 (1:1) films with different ratios of $\mathrm{YCl}_{3}$ are shown in Supplementary Fig. 14. The Y $3 d_{3 / 2}$ peak at $160.4 \mathrm{eV}$ obviously increases with yttrium incorporation in the films. The $\mathrm{Y} / \mathrm{Pb}$ ratios on the surfaces of the films derived from XPS spectra are shown in Fig. 4e. The $\mathrm{Y} / \mathrm{Pb}$ ratios on the film surfaces are three to seven times higher than the corresponding $\mathrm{Y} / \mathrm{Pb}$ ratios in the precursor solution, indicating that yttrium ions accumulate on the film surfaces or grain boundaries.

We examined possible mechanisms that explain the function of $\mathrm{YCl}_{3}$ in PLQE enhancement. Previous study showed organic ligands of layered perovskites have strong impact on the electron-phonon interaction and thus on PLQE of perovskites ${ }^{41}$. To find out possible impact of $\mathrm{Y}$ incorporation on the electron-phonon interaction, we studied the temperature-dependent PL full-width at half-maximum (FWHM) of PEACl:CsPbBr $(1: 1)$ films with or without $\mathrm{YCl}_{3}$, which is a well-established method to derive an electron-phonon interaction ${ }^{41-43}$. As shown in Supplementary Fig. 15, the PL FWHMs of samples with and without $\mathrm{YCl}_{3}$ show the same trend at varied temperatures, which indicates that $\mathrm{YCl}_{3}$ does not notably change charge-phonon interaction strength. We did notice that addition of $\mathrm{YCl}_{3}$ reduced FWHM of PL spectra at all temperatures. This result implies $\mathrm{YCl}_{3}$ can reduce the nonradiative recombination centers in PEACl:CsPbBr ${ }_{3}(1: 1)$ films ${ }^{41}$, which is confirmed by more than two times longer PL decay lifetime after adding $\mathrm{YCl}_{3}$, as shown in Supplementary Fig. 16. Simulation was performed in $\mathrm{CsPbBr}_{3}$ perovskite incorporated with $\mathrm{YCl}_{3}$ to find out why $\mathrm{Y}^{3+}$ ions reduce nonradiative charge recombination. Based on the model, the way of $\mathrm{Y}^{3+}$ incorporation in $\mathrm{CsPbBr}_{3}$ is most likely by replacing $\mathrm{Pb}^{2+}$ and simultaneously forming a $\mathrm{Cs}^{+}$vacancy to compensate the charge difference. As shown in Fig.4f, density function theory calculation reveals $\mathrm{Y}^{3+}$ incorporation increases the bandgap of perovskite, as $\mathrm{Y}$ $4 d$ level is well above the $\mathrm{Pb} 6 p$ states and the $\mathrm{Cl} 3 p$ states are slightly lower than $\mathrm{Br} 3 p$ states. Therefore, the yttrium incorporated region at the shell of the grains impose an energy barrier, which confines charge carriers inside the grains, as is illustrated in Fig. $4 \mathrm{~b}$. This charge confinement minimizes nonradiative recombination that generally occurs at grain boundaries and surfaces due to local large density of defects.

\section{Discussion}

In conclusion, we demonstrated sky-blue PeLED with EQE of $11 \%$ and blue PeLED with EQE of $4.8 \%$, by overcoming the limitation from the low PLQE of wide-bandgap perovskites. The combination of layered perovskite formation and $\mathrm{YCl}_{3}$ incorporation enhances the PLQE from $1.1 \%$ to $49.7 \%$, which also enhance the material stability by suppressing ion migration. This study reveals that the low PLQE in most Cl:Br-based perovskites films limits the LED efficiency. Further research to improve the PLQE of wide-bandgap perovskite films to $100 \%$ should increase the EQE of sky-blue-emission PeLEDs to over $20 \%$. 


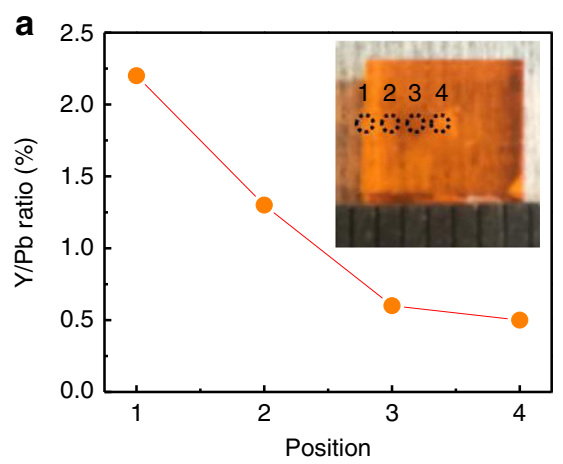

b

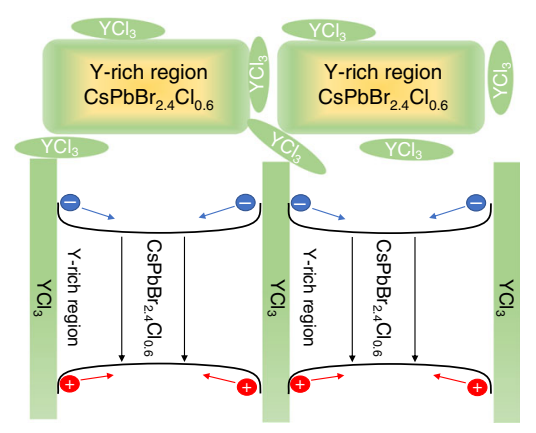

C

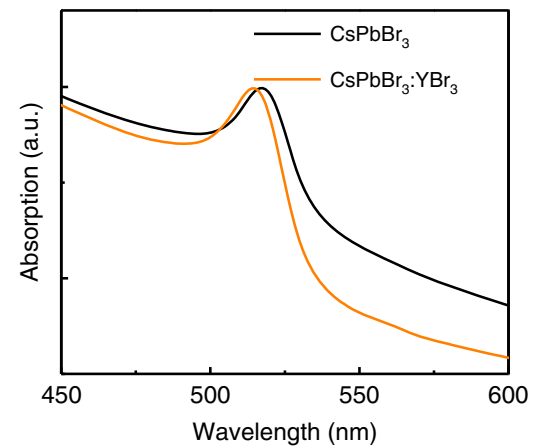

d
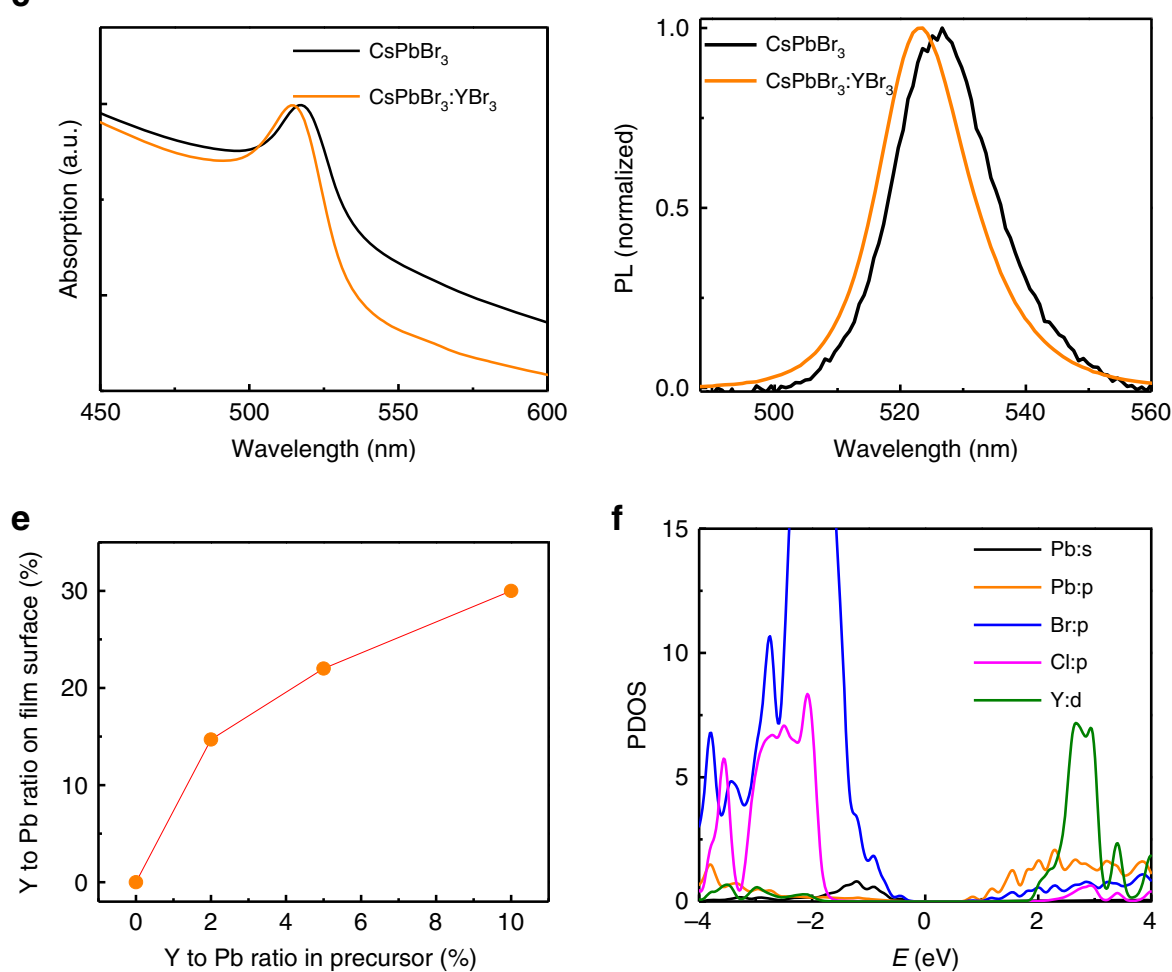

Fig. 4 Mechanism of PLQE enhancement by $\mathbf{Y C l}_{\mathbf{3}}$. a ICP measurement results that show the $\mathrm{Y} / \mathrm{Pb}$ ratios in the different locations of the crystal. The crystal (inset) was cleaved into thin pieces (1-4) and $\mathrm{Y} / \mathrm{Pb}$ ratio of each piece was measured. $\mathbf{b}$ Schematic illustration of the yttrium gradient distribution in the $\mathrm{CsPbBr}_{3}: \mathrm{PEACl}$ (1:1) film and its function in increase the bandgap around the grain surface. Absorption (c) and $\mathrm{PL}$ (d) of $\mathrm{CsPbBr}_{3}$ films with or without $\mathrm{YBr}$. e XPS measurement results that show the $\mathrm{Y} / \mathrm{Pb}$ ratios on the surface of $\mathrm{CsPbBr}_{3}: \mathrm{PEACl}$ (1:1) films with different $\mathrm{YCl} 3 \mathrm{ratios}$ of $0 \%, 2 \%, 5 \%$, and $10 \%$. The $\mathrm{Y} / \mathrm{Pb}$ ratios on the film surface is higher than the corresponding $\mathrm{Y} / \mathrm{Pb}$ ratios in the precursor solutions. $\mathrm{f} D \mathrm{DT}$-calculated $\mathrm{PDOS}$ of $\mathrm{Cs}_{\mathrm{PbBr}}$ with $\mathrm{YCl} \mathrm{C}_{3}$.

\section{Methods}

Material and solution preparation. $\mathrm{PEACl}$ was synthesized by reacting equal molar of phenethylamine (Sigma) with hydrochloric acid solution (Alfa Aesar 37\%) under nitrogen at ice bath for $2 \mathrm{~h}$ with stirring. After reaction, the white precipitate $\mathrm{PEACl}$ was recovered by rotary evaporation at $80^{\circ} \mathrm{C}$ and recrystallized by diethyl ether for three times. The PEACl powders were finally collected and dried at room temperature in a vacuum oven for $24 \mathrm{~h}$. $\mathrm{CsPb}, \mathrm{PbBr}$, and $\mathrm{YCl}_{3}$ were purchased from Sigma Aldrich and TPBI was purchased from Lemtec. The perovskite precursor solution was prepared by mixing $\mathrm{CsPbBr}$ solution, $\mathrm{PEACl}$ solution, and $\mathrm{YCl}_{3}$ solution with desired ratios. $\mathrm{Cs} \mathrm{PbBr}_{3}$ solution $(0.25 \mathrm{M})$ was prepared by mixing $106 \mathrm{mg} \mathrm{CsBr}$ and $183.5 \mathrm{mg} \mathrm{PbBr}_{2}$ in $2 \mathrm{~mL}$ dimethylsulfoxide (DMSO). The $\mathrm{CsPbBr} \mathrm{Pb}_{3}$ solution was filtered before using. PEACl solution $(1 \mathrm{M})$ was prepared by mixing $158 \mathrm{mg} \mathrm{PEACl}$ in $1 \mathrm{~mL}$ DMSO. $\mathrm{YCl}_{3}$ solution $(0.25 \mathrm{M})$ was prepared by mixing $49 \mathrm{mg} \mathrm{YCl}_{3}$ in $1 \mathrm{~mL}$ DMSO.

Perovskite LED fabrication. The indium tin oxide (ITO)-coated glass substrates were sequentially cleaned in detergent, distilled water, acetone, and isopropanol by sonication. The cleaned substrates were treated in UV ozone for $15 \mathrm{~min}$. The, PEDOT:PSS (AI 4083) aqueous solution was spin-coated at 3500 round per minute (RPM) for $40 \mathrm{~s}$ and baked at $125^{\circ} \mathrm{C}$ for $30 \mathrm{~min}$ in ambient air. After that, the substrates were transferred into a nitrogen-filled glovebox and perovskite solution was spin-coated on top of substrate at 4000 RPM for $40 \mathrm{~s}$. Five hundred microliters of chloroform was poured onto perovskite film $20 \mathrm{~s}$ after the spin coating started. The perovskite films were annealed at $90^{\circ} \mathrm{C}$ for $20 \mathrm{~min}$. Finally, the fabrication of PeLEDs was completed by thermally evaporating TPBI $(40 \mathrm{~nm}), \operatorname{LiF}(<1 \mathrm{~nm})$, and $\mathrm{Al}(100 \mathrm{~nm})$ electrode. The device area was $0.10 \mathrm{~cm}^{2}$ as defined by the overlapping area of the ITO and $\mathrm{Al}$ electrode.

Perovskite film and device characterizations. Absorption and PL spectra were measured by Evolution 201 UV-Visible Spectrophotometer and iHR320 Photoluminescence Spectroscopy, respectively. SEM image was measured by FEI Helios 600 system. XPS was measured by Kratos Axis Ultra DLD X-ray Photoelectron Spectrometer. The surface compositions of samples were analyzed by the Processing software on the system. The current $(J)$-voltage $(V)$-luminance characterizations of the devices were performed in $\mathrm{N}_{2}$ glovebox without encapsulation. A Keithley 2400 source meter was used to measure the $J-V$ data from $0 \mathrm{~V}$ to $7 \mathrm{~V}$ with a voltage scanning speed around $0.2 \mathrm{~V} \mathrm{~s}^{-1}$. The luminance of the device was recorded simultaneously by Konica Minolta, LS-160, or a calibrated silicon photodiode (Hamamatsu, S2387 $1010 \mathrm{R}$ ) with an area of $10 \mathrm{~mm} \times 10 \mathrm{~mm}$. The photodiode was placed on top of the LED in close contact to collect light. The EQE was calculated using Lambertian profile and the obtained electroluminescence spectrum. 
Photoluminescence quantum efficiencies were measured by following previous established method ${ }^{44}$. An integrating sphere (Labsphere QE sphere) was connected with a PL spectrometer (Ocean Optics QEpro) by an optical fiber. A continuous wave $403 \mathrm{~nm}$ laser was used to excite the sample. The incident laser intensity was changed by neutral density filters and the intensity was measured by a power meter (Newport 843R). The samples for PLQE measurement were encapsulated and the measurement was performed in air at room temperature. The PLQE results were confirmed by our collaborator at Xi'an Jiao Tong University (Supplementary Fig. 17). Edinburgh FLS980 fluorescence spectrometer system was used in collaborator's PLQE measurement. The Edinburgh FLS980 fluorescence spectrometer has an integrating sphere with a diameter of $150 \mathrm{~mm}$. The excitation light was a monochromatic $365 \mathrm{~nm}$ light and the intensity is $0.5 \mathrm{~mW} \mathrm{~cm}-2$.

ICP mass spectrometry was carried out using a Thermo Element XR-Optical laser ablation source with a double focusing magnet sector. Standards were prepared from THERMO-54 and MSPB calibration standards from Inorganic Ventures and calibration curves were produced for yttrium $(\mathrm{Y})$ to lead $(\mathrm{Pb})$ from 25 parts per trillion to 500 parts per billion. $\mathrm{CsPbBr}_{3}$ with $2 \% \mathrm{YCl}_{3}$ crystals were grown by inverse-temperature crystallization method, which has been widely used to grow high-quality perovskite crystals. Four surfaces of the crystals polished by sand paper to expose the crystal internal. Then the crystal was cleaved into about 1 $\mathrm{mm}$ thin piece by using operation blades. To prevent contamination, each sample was sealed in different vials and the blade was carefully cleaned after each cutting. The samples were then dissolved in $1 \mathrm{~mol} \% \mathrm{HNO}_{3}$ (prepared from Fisher Chemical Nitric Acid TraceMetal Grade and NERL $18 \mathrm{M} \Omega$ water). The solutions were then further diluted with $18 \mathrm{M} \Omega$ water to reach a concentration within the range of the calibration curve. Solutions were injected into the nebulizer and injected into the instrument for analysis. Each sample was followed by a washing period with $1 \mathrm{~mol} \% \mathrm{HNO}_{3}$ in $18 \mathrm{M} \Omega$ water before the next sample. Using the calibration curves produced, the ratio of yttrium $(\mathrm{Y})$ to lead $(\mathrm{Pb})$ was determined.

Time-resolved photoluminescence (TRPL) measurement was performed on a Horiba DeltaPro fluorescence lifetime system. The excitation was provided by a DeltaDiode (DD-405) pulse laser diode with a wavelength of $404 \mathrm{~nm}$. The TRPL curves were fitted to a monoexponential rate law:

$$
y=A \exp \left(-\frac{t}{\tau}\right)+y_{0}
$$

where $A$ is the relative amplitudes and $\tau$ is the lifetimes. The samples for TRPL measurements were perovskite films deposited on glass substrates without HTL. The fluence of TRPL excitation laser and the generated carrier density were $2.0 \mathrm{~nJ} \mathrm{~cm}-2$ and $4.07 \times 10^{14} \mathrm{~cm}^{-3}$, respectively.

Temperature-dependent conductivity measurement for deriving ion migration activation energy was performed in a Lakeshore probe station under a vacuum of $10^{-4} \mathrm{pa}^{31,32}$. The samples were placed on a copper plate with its temperature being controlled by a heater and injected liquid $\mathrm{N}_{2}$. A Keithley 2400 was used for applying voltage bias and measuring the current. The samples for conductivity measurement have lateral Au electrodes on perovskite film. Perovskite films were first fabricated on glass substrates by following the above-mentioned method. Then, $50 \mathrm{~nm}$ Au electrodes with spacing of $50 \mu \mathrm{m}$ were thermally deposited using a mask. Ion activation energy can be derived by fitting the conductivity curve with Nernst-Einstein equation:

$$
\sigma(T)=\frac{\sigma_{0}}{T} \exp \left(-\frac{E_{\mathrm{A}}}{k_{\mathrm{B}} T}\right)
$$

where $\sigma$ is the conductivity, $T$ is the temperature, $E_{\mathrm{A}}$ is the activation energy, and $k_{\mathrm{B}}$ is the Boltzmann constant.

Transient absorption experiment was conducted with a $45 \mathrm{fs}, 4 \mathrm{~mJ}, 800 \mathrm{~nm}$ Coherent Libra with a $1 \mathrm{kHz}$ repetition rate. Single-frequency $400 \mathrm{~nm}$ pump pulses were generated by the second harmonic generation through a barium borate crystal. The remaining $800 \mathrm{~nm}$ component was filtered by two $400 \mathrm{~nm}$ dielectric mirrors. The pulse energies were controlled by a neutral density filter. Continuum probe pulses were generated in a sapphire window and relayed to the sample with reflective optics. The spot size of the probe was adjusted to match the $200 \mu \mathrm{m}$ spot size of the pump. Signal detection was accomplished with a high-speed complementary metal-oxide semiconductor array detector that is synchronized to the laser system.

Temperature-dependent PL spectrum measurement was performed by using iHR320 Photoluminescence Spectroscopy and a portal LINKAM thermal stage. The FWHM of PL spectra was fitted by the following equation to derive an electron-phonon interaction strength ${ }^{41-43}$ :

$$
\Gamma(T)=\Gamma_{0}+\frac{\Gamma_{1}}{\exp \left(\frac{\hbar \omega_{1}}{k_{\mathrm{B}} T}\right)-1}
$$

where $\Gamma_{0}$ is temperature-independent inhomogeneous broadening term, which arises from scattering due to disorder and imperfections. $\Gamma_{1}$ represents the electron-phonon coupling strength, primarily contributed by longitudinal optical phonon scattering. $\omega_{1}$ is the homopolar phonon frequency, which is $133 \mathrm{~cm}^{-1}$ for $\mathrm{Pb}-\mathrm{Br}-\mathrm{Pb}$ stretch, based on previous report. The electron-phonon coupling strengths $\left(\Gamma_{1}\right)$ in Supplementary Fig. 15 were fitted to be $0.032 \mathrm{eV}$ and $0.035 \mathrm{eV}$ in $\mathrm{PEACl}: \mathrm{CsPbBr}_{3}(1: 1)$ films with or without $\mathrm{YCl}_{3}$, respectively. $\Gamma_{0}$ was reduced from $0.093 \mathrm{eV}$ in $\mathrm{PEACl}: \mathrm{CsPbBr}_{3}(1: 1)$ film to $0.086 \mathrm{eV}$ in $\mathrm{PEACl}_{\mathrm{CsPbBr}}(1: 1)$ film with $\mathrm{YCl}_{3}$.

Density functional theory calculations were performed using the VASP code $e^{45,46}$ with projector augmented-wave ${ }^{47}$ potentials. The $\mathrm{PBE}^{48}$ exchange-correlation functional and a kinetic energy cutoff of $300 \mathrm{eV}$ were employed. Spin-orbit coupling was included in the calculations due to the heavy $\mathrm{Pb}$ atoms. To model the surface passivation, we built a $\mathrm{CsPbBr}$ slab with the top surface layer covered by $\left[\mathrm{YCl}_{6}\right]$ octahedra. The cell size is $1.2 \mathrm{~nm} \times 1.2 \mathrm{~nm} \times 2.3 \mathrm{~nm}$. A vacuum thickness of $15 \AA$ was used to eliminate spurious periodic interactions between the slabs. The bottom two layers of $\left[\mathrm{PbBr}_{6}\right]$ octahedra were fixed at their relaxed bulk positions, while all other atoms were relaxed with a force tolerance of $0.01 \mathrm{eV} \AA^{-1}$. A $\Gamma$-centered $k$ mesh of $4 \times 4 \times 1$ was used to sample the Brillouin zone.

\section{Data availability}

The data that support the findings of this study are available from the corresponding author upon reasonable request. The source data underlying Figs. 1a, d, 2c-i, 3a-e, and $4 \mathrm{a}, \mathrm{c}-\mathrm{f}$ are provided as a Source Data.

Received: 28 October 2019; Accepted: 13 November 2019; Published online: 10 December 2019

\section{References}

1. Kovalenko, M. V., Protesescu, L. \& Bodnarchuk, M. I. Properties and potential optoelectronic applications of lead halide perovskite nanocrystals. Science 358, 745-750 (2017).

2. Stranks, S. D. \& Snaith, H. J. Metal-halide perovskites for photovoltaic and light-emitting devices. Nat. Nanotechnol. 10, 391 (2015).

3. Cao, Y. et al. Perovskite light-emitting diodes based on spontaneously formed submicrometre-scale structures. Nature 562, 249 (2018).

4. Lin, K. et al. Perovskite light-emitting diodes with external quantum efficiency exceeding 20 per cent. Nature 562, 245 (2018).

5. Chiba, T. et al. Anion-exchange red perovskite quantum dots with ammonium iodine salts for highly efficient light-emitting devices. Nat. Photonics 12, 681 (2018).

6. Zhao, B. et al. High-efficiency perovskite-polymer bulk heterostructure lightemitting diodes. Nat. Photonics 12, 783-789 (2018).

7. Gong, X. et al. Highly efficient quantum dot near-infrared light-emitting diodes. Nat. Photonics 10, 253 (2016).

8. $\mathrm{Xu}, \mathrm{W}$. et al. Rational molecular passivation for high-performance perovskite light-emitting diodes. Nat. Photonics 13, 418-424 (2019).

9. Wang, N. et al. Perovskite light-emitting diodes based on solution-processed self-organized multiple quantum wells. Nat. Photonics 10, 699 (2016).

10. Cho, H. et al. Overcoming the electroluminescence efficiency limitations of perovskite light-emitting diodes. Science 350, 1222-1225 (2015).

11. Luo, J. et al. Efficient and stable emission of warm-white light from lead-free halide double perovskites. Nature 563, 541 (2018).

12. Xiao, Z. et al. Efficient perovskite light-emitting diodes featuring nanometresized crystallites. Nat. Photonics 11, 108 (2017).

13. Wang, $\mathrm{H}$. et al. Trifluoroacetate induced small-grained $\mathrm{Cs} \mathrm{PbBr} 3$ perovskite films result in efficient and stable light-emitting devices. Nat. Commun. 10, 665 (2019).

14. Shang, Y., Li, G., Liu, W. \& Ning, Z. Quasi-2D inorganic CsPbBr3 perovskite for efficient and stable light-emitting diodes. Adv. Funct. Mater. 28, 1801193 (2018).

15. Liu, Y. et al. Efficient blue light-emitting diodes based on quantum-confined bromide perovskite nanostructures. Nat. Photonics 13, 760-764 (2019).

16. $\mathrm{Li}, \mathrm{Z}$. et al. Modulation of recombination zone position for quasi-twodimensional blue perovskite light-emitting diodes with efficiency exceeding 5\%. Nat. Commun. 10, 1027 (2019).

17. Jiang, Y. et al. Spectra stable blue perovskite light-emitting diodes. Nat. Commun. 10, 1868 (2019).

18. Vashishtha, P., Ng, M., Shivarudraiah, S. B. \& Halpert, J. E. High efficiency blue and green light-emitting diodes using Ruddlesden-Popper inorganic mixed halide perovskites with butylammonium interlayers. Chem. Mater. 31, 83-89 (2018).

19. Abdi-Jalebi, M. et al. Maximizing and stabilizing luminescence from halide perovskites with potassium passivation. Nature 555, 497 (2018).

20. Braly, I. L. et al. Hybrid perovskite films approaching the radiative limit with over $90 \%$ photoluminescence quantum efficiency. Nat. Photonics 12, 355-361 (2018).

21. Shi, X. B. et al. Optical energy losses in organic-inorganic hybrid perovskite light-emitting diodes. Adv. Opt. Mater. 6, 1800667 (2018).

22. Xing, J. et al. Color-stable highly luminescent sky-blue perovskite lightemitting diodes. Nat. Commun. 9, 3541 (2018). 
23. Ban, M. et al. Solution-processed perovskite light emitting diodes with efficiency exceeding $15 \%$ through additive-controlled nanostructure tailoring. Nat. Commun. 9, 3892 (2018).

24. Yang, X. et al. Efficient green light-emitting diodes based on quasi-twodimensional composition and phase engineered perovskite with surface passivation. Nat. Commun. 9, 570 (2018).

25. Dohner, E. R., Jaffe, A., Bradshaw, L. R. \& Karunadasa, H. I. Intrinsic whitelight emission from layered hybrid perovskites. J. Am. Chem. Soc. 136, 13154-13157 (2014).

26. Li, G. et al. Highly efficient perovskite nanocrystal light-emitting diodes enabled by a universal crosslinking method. Adv. Mater. 28, 3528-3534 (2016).

27. Lin, Y. et al. Excess charge-carrier induced instability of hybrid perovskites Nat. Commun. 9, 4981 (2018).

28. Slotcavage, D. J., Karunadasa, H. I. \& McGehee, M. D. Light-induced phase segregation in halide-perovskite absorbers. ACS Energy Lett. 1, 1199-1205 (2016).

29. $\mathrm{Li}, \mathrm{W}$. et al. Phase segregation enhanced ion movement in efficient inorganic CsPbIBr2 solar cells. Adv. Energy Mater. 7, 1700946 (2017).

30. Barker, A. J. et al. Defect-assisted photoinduced halide segregation in mixedhalide perovskite thin films. ACS Energy Lett. 2, 1416-1424 (2017).

31. Yuan, Y. et al. Electric-field-driven reversible conversion between Methylammonium lead triiodide perovskites and lead iodide at elevated temperatures. Adv. Energy Mater. 6, 1501803 (2016).

32. Xing, J. et al. Ultrafast ion migration in hybrid perovskite polycrystalline thin films under light and suppression in single crystals. Phys. Chem. Chem. Phys. 18, 30484-30490 (2016).

33. Mizusaki, J., Arai, K. \& Fueki, K. Ionic conduction of the perovskite-type halides. Sol. State Ion. 11, 203-211 (1983).

34. Lin, Y. et al. Suppressed ion migration in low-dimensional perovskites. ACS Energy Lett. 2, 1571-1572 (2017).

35. Lin, Y. et al. Unveiling the operation mechanism of layered perovskite solar cells. Nat. Commun. 10, 1008 (2019).

36. Ma, D. et al. Single-crystal microplates of two-dimensional organic-inorganic lead halide layered perovskites for optoelectronics. Nano Res. 10, 2117-2129 (2017).

37. Bhaumik, S. et al. Highly stable, luminescent core-shell type methylammonium-octylammonium lead bromide layered perovskite nanoparticles. Chem. Commun. 52, 7118-7121 (2016).

38. Yuan, M. et al. Perovskite energy funnels for efficient light-emitting diodes. Nat. Nanotechnol. 11, 872 (2016).

39. Saidaminov, M. I. et al. High-quality bulk hybrid perovskite single crystals within minutes by inverse temperature crystallization. Nat. Commun. 6, 7586 (2015).

40. Dirin, D. N., Cherniukh, I., Yakunin, S., Shynkarenko, Y. \& Kovalenko, M. V. Solution-Grown $\mathrm{CsPbBr} 3$ Perovskite single crystals for photon detection. Chem. Mater. 28, 8470-8474 (2016).

41. Gong, X. et al. Electron-phonon interaction in efficient perovskite blue emitters. Nat. Mater. 17, 550 (2018).

42. Guo, Z., Wu, X., Zhu, T., Zhu, X. \& Huang, L. Electron-phonon scattering in atomically thin 2D perovskites. ACS Nano 10, 9992-9998 (2016).

43. Wright, A. D. et al. Electron-phonon coupling in hybrid lead halide perovskites. Nat. Commun. 7, 11755 (2016).

44. de Mello, J. C., Wittmann, H. F. \& Friend, R. H. An improved experimental determination of external photoluminescence quantum efficiency. Adv. Mater. 9, 230-232 (1997)

45. Kresse, G. \& Furthmüller, J. Efficient iterative schemes for ab initio totalenergy calculations using a plane-wave basis set. Phys. Rev. B 54, 11169 (1996).
46. Kresse, G. \& Furthmüller, J. Efficiency of ab-initio total energy calculations for metals and semiconductors using a plane-wave basis set. Comput. Mater. Sci. 6, 15-50 (1996)

47. Blöchl, P. E. Projector augmented-wave method. Phys. Rev. B 50, 17953 (1994).

48. Perdew, J. P., Burke, K. \& Ernzerhof, M. Generalized gradient approximation made simple. Phys. Rev. Lett. 77, 3865-3868 (1996).

\section{Acknowledgements}

We acknowledge support for the device work from National Science Foundation under award DMR-1903981. We acknowledge support for the mechanism study including computation from the Center for Hybrid Organic Inorganic Semiconductors for Energy (CHOISE), an Energy Frontier Research Center funded by the DOE Office of Basic Energy Sciences, Office of Science. A.M. acknowledges support for the transient absorption characterizations from the National Science Foundation grant CHE-1763207

\section{Author contributions}

J.H. and Q.W. conceived the idea. Q.W. fabricated the devices and performed the deviceefficiency measurement. Z.Y. conducted photoluminescence quantum yield measurement. N.Z. and A.M. conducted transient absorption measurement. J.Z., Y.D., and X.X. conducted X-ray diffraction measurement. P.R. conducted ICP measurement. X.W. and Y.Y. conducted the simulation. Q.W. and J.H. wrote the paper.

\section{Competing interests}

The authors declare no competing interests.

\section{Additional information}

Supplementary information is available for this paper at https://doi.org/10.1038/s41467 019-13580-w.

Correspondence and requests for materials should be addressed to J.H.

Peer review information Nature Communications thanks the anonymous reviewer(s) for their contribution to the peer review of this work.

Reprints and permission information is available at http://www.nature.com/reprints

Publisher's note Springer Nature remains neutral with regard to jurisdictional claims in published maps and institutional affiliations.

pen Access This article is licensed under a Creative Commons Attribution 4.0 International License, which permits use, sharing, adaptation, distribution and reproduction in any medium or format, as long as you give appropriate credit to the original author(s) and the source, provide a link to the Creative Commons license, and indicate if changes were made. The images or other third party material in this article are included in the article's Creative Commons license, unless indicated otherwise in a credit line to the material. If material is not included in the article's Creative Commons license and your intended use is not permitted by statutory regulation or exceeds the permitted use, you will need to obtain permission directly from the copyright holder. To view a copy of this license, visit http://creativecommons.org/ licenses/by/4.0/.

(C) The Author(s) 2019 